\title{
Hydrodynamic effects in interacting Fermi electron jets
}

\author{
Alexander O. Govorov ${ }^{1}$ and Jean J. Heremans ${ }^{1}$ \\ ${ }^{1}$ Department of Physics and Astronomy, \\ and The Nanoscale and Quantum Phenomena Institute, \\ Ohio University, Athens, Ohio 45701
}

(Dated: September 2, 2018)

\begin{abstract}
We theoretically study hydrodynamic phenomena originating from electron-electron collisions in a two-dimensional Fermi system. We demonstrate that an electron beam sweeping past an aperture creates a pumping effect, attracting carriers from this aperture. This pumping effect originates from the specific electric potential distribution induced by the injected electrons. In the regions nearby the main stream of injected electrons, a positive potential is induced by the injected electrons. Thus, the normally repulsive Coulomb interaction leads to an attractive force in the Fermi system. This quantum pumping mechanism in a Fermi system differs qualitatively from the Bernoulli pumping effect in classical liquids. We also discuss possible experimental realizations.
\end{abstract}

PACS numbers: 73.23.Ad; 73.63.Hs 
Electron-electron (e-e) scattering in a degenerate electron gas forms one of the central concepts of Fermi-liquid theory ${ }^{1.2}$. Electron states with energy slightly above the Fermi surface are not stationary, since they decay accompanied by the creation of electron-hole pairs in the Fermi sea ${ }^{1}$. The total momentum in the e-e scattering process is conserved, and hence the e-e collisions do not in the main affect the conductivity. At the same time, the e-e collisions play an important role in experiments which involve the phase coherence time of the electron ${ }^{3.4}$, and in physical effects implicating hydrodynamics ${ }^{5.6 .7 .8}$. At low temperatures, the e-e collision processes slow down due to Fermi statistics $\frac{9.10 .11}{2}$, leading, in the ideal two dimensional electron system (2DES), to the inverse e-e lifetime rapidly decreasing upon lowering the temperature $T^{9.10}$ :

$$
\frac{1}{\tau_{e e}}=\frac{\left(k_{B} T\right)^{2}}{h E_{F}}\left[\ln \frac{E_{F}}{k_{B} T}+\ln \frac{4}{a_{0}^{*} k_{F}}+1\right],
$$

where $h, E_{F}$, and $v_{F}$ are the Planck's constant, the Fermi energy, and the Fermi velocity, respectively; $a_{0}^{*}$ denotes the effective Bohr radius. To render hydrodynamic effects in 2DESs observable, e-e collisions should occur more often than impurity scattering events. Mathematically, this condition is obvious: $l_{e e}<l_{p}$, where $l_{e e}=v_{F} \tau_{e e}$ is the electron mean free path related to e-e scattering and $l_{p}$ is the momentum relaxation mean free path. The above condition is satisfied in typical high-mobility GaAs/GaAlAs heterostructures, in the temperature range $T \sim 5-35 K$ (Fig. 1a) ${ }^{12}$. Therefore, the hydrodynamic effects should be accessible in experimental low-temperature studies. Here we describe a new hydrodynamic phenomenon in a low-temperature electron plasma which occurs when an electron beam is injected from a narrow aperture into a 2DES (Fig. 1b). We show that the injected beam creates an unusual potential pattern which can result in a hydrodynamic pumping effect. This pumping effect exists in mesoscopic structures where the injected electrons experience just few e-e collisions. In contrast to the Bernoulli effect in classical liquids, the predicted quantum pumping behaves linearly in the excitation, and can only be observed in a Fermi system at low temperatures.

Another potential application of the predicted hydrodynamic effect concerns measurements of the electron phase coherence time, $\tau_{\phi}$. The low-temperature behavior of $\tau_{\phi}$ in nanostructures is a long standing problem. According to the standard theory, e-e scattering becomes very weak at low temperatures and therefore $\tau_{\phi}$ should tend to infinity at zero temperature. However, the experiments demonstrate that $\tau_{\phi}$ saturates to a finite value at 
low temperatures 3.4 . Magnetic-impurity scattering has been implicated amongst others ${ }^{4}$. Yet, e-e scattering itself is a nontrivial problem in mesoscopic structures, where an electron experiences scattering by boundaries as well as impurities. For example, it is accepted that in diffusive metals, the formula $1 / \tau_{e e} \propto T^{2}$ should be strongly modified due to disorder 11 . Measurements of the hydrodynamic effect predicted in this work can directly reveal the contribution of e-e scattering to the phase coherence time in mesoscopic ballistic structures at low temperatures.

To describe hydrodynamic effects in a stationary electron beam (Fig. 1b) we employ the linearized Boltzmann equation:

$$
\mathbf{v} \frac{\partial f_{1}}{\partial \mathbf{r}}+e \mathbf{E} \frac{\partial f_{0}}{\partial \mathbf{p}}=J\left(f_{1}\right)
$$

where $f_{1}(\mathbf{r}, \mathbf{p})$ is the distribution function of the non-equilibrium electrons, $\mathbf{r}=(x, y)$ and $\mathbf{p}$ are the two-dimensional coordinate and momentum, respectively; $f_{0}(\epsilon)$ is the equilibrium Fermi function, $\mathbf{v}=\mathbf{p} / m$ is the electron velocity, and $\epsilon$ and $m$ are the energy and the effective mass of the electron, respectively; $\mathbf{E}$ is the in-plane electric field, $e=-|e|$ is the electron charge, and $J$ is the collision integral. At low temperature, the non-equilibrium electrons have energies close to the Fermi surface and the function $f_{1}$ can be written as $f_{1}(\mathbf{r} ; \epsilon, \theta)=-\frac{\partial f_{0}}{\partial \epsilon} F(\mathbf{r} ; \theta)$, where $\theta$ is the angle between the velocity and the direction $+x$, with $0<\theta<2 \pi$. Our choice of $(x, y)$ coordinates is indicated in Fig. 1b.

We treat e-e collisions in the relaxation-time approximation. Due to the complexity of the collision integral incorporating the long-range Coulomb interaction, theoretical studies based on the long-range Coulomb collision integral usually include only the first e-e collision event $^{5,14}$. In contrast, the relaxation-time approach allows us to obtain analytical results for an infinite number of collisions and to describe fundamental hydrodynamic effects appearing in the multi-collision regime. Within the relaxation-time approximation, the collision integral describing scattering events in the vicinity of the Fermi surface, takes the form:

$$
J(F)=-\frac{F-\bar{F}-2 \cos \theta \overline{\cos \theta F}-2 \sin \theta \overline{\sin \theta F}}{\tau_{e e}}
$$

where $\bar{F}=\int_{0}^{2 \pi} F(\theta) d \theta / 2 \pi$ and $\tau_{e e}$ is the e-e scattering time. Note that the e-e collision integral (3) conserves both the number of particles and the momentum. In addition, the collision integral (3) assumes an ideal system without disorder and is valid if $l_{e e}<l_{p}$. 
To solve the Boltzmann equation in the half-plane $x>0$ we need to impose the boundary condition (BC) at the line $x=0$. Outside the aperture, the $\mathrm{BC}$ describes the elastic collision of an electron with an ideal border, $F(0, y ; \theta)-F(0, y ; \pi-\theta)=0$. In order to express the injection process, we now introduce the injection function $g(y, \theta)$, nonzero only in the angle intervals $0<\theta<\pi / 2$ and $(3 / 2) \pi<\theta<2 \pi$. It is convenient to consider the $\mathrm{BC}$ separately in two intervals, $-\pi / 2<\theta<\pi / 2$ and $\pi / 2<\theta<(3 / 2) \pi$. In the first angular interval, the outgoing flux of electrons $v_{F} F(\theta)$ should equal the sum of the incoming and the injected fluxes, $v_{F} F(\pi-\theta)+v_{F} g(\theta)$. A similar argument is applied to the second interval. In both intervals, the $\mathrm{BC}$ condenses to a single formula:

$$
F(0, y ; \theta)-F(0, y ; \pi-\theta)=g(y ; \theta)-g(y ; \pi-\theta) .
$$

Importantly, the $\mathrm{BC}$ (44) leaves some freedom in choosing the function $F(0, y ; \theta)$. A general solution of eq. 4 can be written as $F(0, y ; \theta)=w_{1}(y ; \theta)+w_{2}(y ; \theta)$, where the function $w_{1}(\theta)$ is determined by the injection $g(y ; \theta)$, whereas the function $w_{2}(\theta)$ is arbitrary and satisfies the elastic-collision condition $w_{2}(y ; \theta)-w_{2}(y ; \pi-\theta)=0$.

The BCs in this problem are given by the equation 4 at the line $x=0$ and by the condition $F(x \rightarrow \infty) \rightarrow 0$ at infinity. To solve this problem in a rather convenient way, we now consider a symmetric problem, involving the entire two-dimensional plane and two symmetric current sources (two jets) in the vicinity of $\mathbf{r}=0$. Including the two current sources, we write the linearized Boltzmann equation as follows:

$$
\mathbf{v} \frac{\partial F}{\partial \mathbf{r}}-e \mathbf{E v}=J(F)+G(y ; \theta) \delta(x)+G(y ; \pi-\theta) \delta(x),
$$

where the function $G(y ; \theta)$ describes the injection. By exploiting the symmetry of the problem and integrating eq. 5 over $x$ in the vicinity of $x=0$, we determine that the solution of eq. [5 satisfies the necessary BC (4) with $G(\theta)=v_{F} \cos \theta g(\theta)$. Equation (15) can be solved using Fourier transformation,

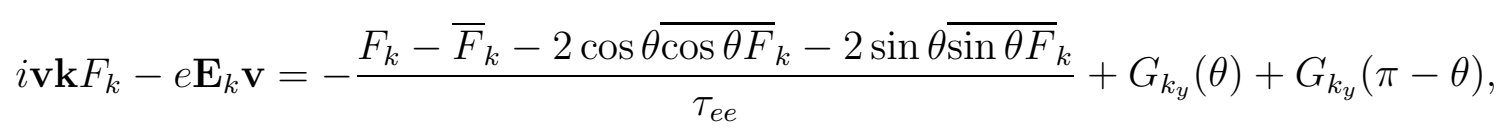

where $F_{\mathbf{k}}(\theta)=\int_{-\infty}^{+\infty} d^{2} \mathbf{r} e^{-i \mathbf{k r}} F(\mathbf{r} ; \theta)$. The electric field $\mathbf{E}$ in eq. [6 originates from the nonequilibrium electrons. We now assume that the heterostructure containing the 2DES is 
covered by a top metallic gate, with the distance between the 2DES and the top gate $d$ smaller than any lateral size of the system. This condition does not unduly restrict experimental situations. Imposing this condition ensures that the potential $\phi$ in the plane of $2 \mathrm{D}$ plasma is proportional to the non-equilibrium $2 \mathrm{DES}$ density $\delta n(\mathbf{r})$. Thus, we can write $\phi(\mathbf{r})=\left(4 \pi e d / \epsilon_{s}\right) \delta n(\mathbf{r})$, where $\epsilon_{s}$ denotes the dielectric constant of the semiconductor $\frac{8.15}{}$. After Fourier transformation, we obtain $\mathbf{E}_{k}=-i \mathbf{k} \phi_{k}=-i \mathbf{k}\left(4 \pi e d / \epsilon_{s}\right) \delta n_{k}$. Simultaneously, the electron density is expressed through the function $\bar{F}, \delta n_{k}=\bar{F}_{k} D_{2 D}$, where $D_{2 D}=m / \pi \hbar^{2}$ represents the $2 \mathrm{D}$ density of states. By manipulating eq. [6 and integrating over angles, we obtain a closed system of equations for $\bar{F}_{k}, \overline{\cos \theta F}_{k}$, and ${\overline{\sin } \theta F_{k}}_{k}$. The 2DES density can be determined by the functions

$$
\begin{gathered}
\bar{F}_{\mathbf{k}}=\frac{I_{1} \frac{2 k_{0}}{k^{2}}\left(k_{0} W_{0}-1\right)+I_{2}}{\left(1+4 d / a_{0}^{*}\right)\left(1-k_{0} W_{0}\right)}, \\
W_{0}(k)=\frac{1}{\sqrt{k^{2}+k_{0}^{2}}}, \quad I_{1}=\int_{0}^{2 \pi} G_{k_{y}}(\theta) \frac{d \theta}{\pi v_{F}}, \quad I_{2}(\mathbf{k})=\int_{0}^{2 \pi} \frac{G_{k_{y}}(\theta)+G_{k_{y}}(\pi-\theta)}{i k \cos (\theta-\alpha)+k_{0}} \frac{d \theta}{2 \pi v_{F}},
\end{gathered}
$$

where $\tan \alpha=k_{y} / k_{x}$ and $k_{0}=\left(v_{F} \tau_{e e}\right)^{-1}=1 / l_{e e}$. We also derive analytical expressions for the current densities, $j_{x}(\mathbf{k})=v_{F} D_{2 D} \overline{\cos \theta F}_{k}$ and $j_{y}(\mathbf{k})=v_{F} D_{2 D}{\overline{\sin \theta F_{k}}}_{k}$.

For the injection function, we choose

$$
G(y, \theta)=G_{\max } e^{-\frac{y^{2}}{L^{2}}} \frac{\Theta\left(\theta_{0}-|\theta|\right)}{2 \theta_{0}},
$$

with $\Theta(\theta)=1$ if $\theta>0$ and 0 otherwise. The parameters $L$ and $\theta_{0}$ describe the spatial width of the aperture and the width of the angular distribution of injected electrons, respectively. The parameter $G_{\max }$ is proportional to the injected current density in the middle of the aperture. Using the injection function, $G_{\max }$ can be related to the total injected current, by $I=|e| D_{2 D} L /(2 \sqrt{\pi}) G_{\max }$. Typically, the resistance of the injecting aperture exceeds the resistance of the leads and thus the potential drop across the aperture, $\Delta V$, dominates the potential applied to the system. Using the Landauer-Büttiker formalism, we can write $I=\left(2 e^{2} / h\right) N \Delta V^{\underline{16}}$, where $N$ represents the number of conducting modes in the aperture. Although quantization of the conductance is not necessary for the observation of the hydrodynamic effect ( $N$ can be large), the Landauer-Büttiker formalism forms a convenient framework to link the experimental variables, $L, N, \Delta V$ and $I$. By combining the above 
equations, we obtain: $G_{\max }=4 \sqrt{\pi}|e| N /\left(h L D_{2 D}\right) \Delta V$. In addition, it was assumed that the injected electrons possess energies in the vicinity of the Fermi surface.

Numerical results for the non-equilibrium density distribution follow from the reverse transformation, $\delta n=D_{2 D} \int \exp (i \mathbf{k r}) \bar{F}_{k} d \mathbf{k} /(2 \pi)^{2}$. Figure 2 shows that $\delta n$ is large and positive inside the ballistic beam, as expected. But $\delta n$ is depressed to negative values in regions nearby the main stream of injected electrons. The negative $\delta n$ corresponds to depletion. In Fig. 3, we schematically show the streamlines associated with injection. Interestingly, in the regions adjacent to the main stream, the currents flow towards the beam. Qualitatively, we can understand this behavior in terms of e-e scattering: the injected electrons create an effective pressure and scatter the background Fermi-sea electrons toward the right. Numerical calculations show that this happens not in the main stream, where the density of excess electrons is high, but rather on the sides of the main stream. The currents flowing toward the main stream tend to compensate for the lack of electrons.

The electron depletion in the vicinity of the injected beam can lead to carrier pumping toward this beam. The potential induced by the non-equilibrium electrons can be expressed as $\phi(\mathbf{r})=\left(4 \pi e d / \epsilon_{s}\right) \delta n(\mathbf{r})$, reflecting the fact that a local net charge will lead to a local potential of the same sign. If a detector aperture is situated in the region of electron depletion $(\delta n<0)$, the detector will experience a positive potential (Fig.3). In voltage measurement mode, the detector can be considered a closed reservoir with net zero current. A positive potential denotes a lack of electrons in the detector reservoir, the result of a net pumping of electrons toward the injected beam. We can also consider the pumping in terms of currents. Suppose that we turn on the injected current $I$ at $t=0$. In the region of the detector window, the pumping effect extracts electrons from the detector. After some time, the system reaches steady state, and the net current through the detector window vanishes. Hence a counter current of electrons into the detector must be generated, by a positive potential on the detector lead. If on the contrary current is allowed to flow, electrons will be pumped through the aperture in steady state. To achieve the steady state pumping, we can connect the detector contact to the Fermi sea in the right-hand side, including a resistor to maintain the current at a level sufficiently low for the latter to be regarded as a weak perturbation. In all above cases, the positive potential induced by the hydrodynamic effects in the vicinity of the injected beam, effectively result in a pumping phenomenon.

According to eq. 1, the length $l_{e e}=v_{F} \tau_{e e}$ strongly depends on temperature, leading 
to a temperature dependence of the pumping effect. In the ideal disorderless system, the pumping potential depends on the ratios $L / l_{e e}, x / l_{e e}$, and $y / l_{e e}$ (Fig. 1b). It is revealing to analyze the temperature dependence of the induced electric potential at a fixed position with coordinates $\left(x_{A}, y_{A}\right)$ where a detector window can be located (Fig. 3). Calculations (Fig. 3) indicate that the potential induced by the injected beam at the point $A$ with coordinates $\left(x_{A}=2 \mu m, y_{A}=-2 \mu m\right)$ is negative at low and high temperatures, while for intermediate temperatures, $3-25 K$, the hydrodynamic effects render the potential positive. In this regime, $l_{e e} \sim x_{A} \sim y_{A}$ and electron-electron interaction leads to just a few scattering events in the vicinity of the detector. Therefore, the pumping effect occurs only in the regime of few scattering events. Our calculations show that the pumping voltage increases by decreasing the angle $\theta_{0}$. Hence, better experimental conditions to observe the pumping signal can be found in relatively wide apertures with flared openings, where the collimation effect is typically stronger ${ }^{17}$. In addition, since $\phi \propto \delta n \propto G_{\max } \propto \Delta V \propto I$, the pumping voltage is a linear function of the applied voltage or injected current.

In the case of one-dimensional injection $(L \rightarrow \infty)$, the function $\delta n(x)$ can be obtained from eq. [7 by setting $G_{k_{y}} \propto \delta\left(k_{y}\right)$. The calculated electron density clearly demonstrates the hydrodynamic effect: the Fermi sea depletes in the region $x \sim l_{e e}$ due to the effective pressure created by the injected beam (Fig. 4). This effect can also be applied toward a pumping mechanism involving a quasi-one-dimensional channel featuring an injecting barrier and a detector window, as illustrated in Fig. 4.

It is interesting to compare our results with the hydrodynamics of classical liquids. Of course, the local non-linear equations of fluid mechanics differ qualitatively from the nonlocal kinetic Boltzmann equation. For example, the Bernoulli pumping effect, resulting from the spatial variation of local speed in the moving liquid, is quadratic in terms of fluid speed, hence non-linear. As result the Bernoulli pumping effect remains unchanged if we reverse the current. In the 2DES case discussed in this work, the pumping force is linear in terms of applied voltage or current and changes sign as we reverse the current flow. The linearity is a consequence of the electron Fermi statistics. Indeed, at low temperatures, we can consider the flow equally in terms of electrons or of holes near the Fermi surface. Mathematically, the described linear response originates naturally from the linearized Boltzmann equation. Physically, when the negative voltage is applied to the injecting contact, the injected beam consists of electrons which induce an effective pumping force for the electrons from a detector 
(Fig. 3). When a positive voltage is applied, the injected beam can be treated as a beam of holes near the Fermi surface; these holes now induce an effective pumping force for the holes from the detector and therefore the pumping voltage reverses its sign, along with the applied voltage. The concurrent voltage reversals lead to a linear dependence at low excitations. The linear dependence stands in contrast to classical liquids where currents consist of like particles and the above Fermi-gas description cannot be applied.

To conclude, we have shown that the Coulomb interaction in an injected ballistic beam results in attractive forces which can be exploited toward a pumping effect. The quantum pumping described by us is a peculiar property of a degenerate 2DES and is qualitatively different from the hydrodynamic effects in classical liquids.

We thank Axel Lorke for helpful discussions. This work was supported by NSF (DMR0094055) and by the Condensed Matter and Surface Science Program at Ohio University.

1 E. M. Lifshitz and L. P. Pitaevskii, Statistical Physics, Part 2, (Pergamon Press, N.Y., 1980).

2 L.D. Landau, Zh. Eksp. Teor. Fiz. 30, 1058 (1956) [Sov. Phys. JETP 3, 920 (1956)].

3 P. Mohanty, E. M. Q. Jariwala, and R. A. Webb, Phys. Rev. Lett. 78, 3366 (1997); D. Natelson, R. L. Willett, K. W. West, and L. N. Pfeiffer, ibid 86, 1821 (2001).

4 A. Anthore, F. Pierre, H. Pothier, and D. Esteve, Phys. Rev. Lett. 90, 076806 (2003); F. Pierre and N. O. Birge, ibid 89, 206804 (2002).

5 M. J. M. de Jong and L. W. Molenkamp, Phys. Rev. B 51, 13389 (1995); H. Predel, H. Buhmann, L. W. Molenkamp, R. N. Gurzhi, A. N. Kalinenko, A.I. Kopeliovich and A. V. Yanovsky, Phys. Rev. B 62, 2057 (2000);

6 M. Dyakonov and M. Shur, Phys. Rev. Lett. 71, 2465 (1993).

7 A. M. Song, A. Lorke, A. Kriele, J. P. Kotthaus, W. Wegscheider, and M. Bichler, Phys. Rev. Lett. 80, 3831 (1998).

8 A. O. Govorov, V. M. Kovalev and A. V. Chaplik, JETP Lett. 70, 488 (1999).

9 A. V. Chaplik, Zh. Eksp. Teor. Fiz. 60, 1845 (1971) [Sov. Phys.-JETP 33, 997 (1971)]. 
10 G. F. Giuliani and J. J. Quinn, Phys. Rev. B 26, 4421 (1982).

11 B. L. Altshuler, A. G. Aronov and D. E. Khmelnitsky, J. Phys. C 15, 7367 (1982).

12 For $T$ higher than about $35 K$, the mobility and the momentum relaxation time in GaAsquantum wells fall rapidly due to strong polar LO-phonon scattering, disrupting ballistic transport. This imposes a high- $T$ limit on the observation of the described hydrodynamic effect. For the T-dependence of scattering mechanisms, see e.g. K. Hirakawa and H. Sakaki, Phys. Rev. B 33, 8291 (1986).

13 J. J. Heremans et al., to be published.

14 D. R. S. Cumming and J. H. Davies, Appl. Phys. Lett. 69, 3363 (1996).

15 W. R. Frank, A. O. Govorov, J. P. Kotthaus, and W. Hansen, Phys. Rev. B 55, R1950 (1997).

16 D. A. Wharam et al., J. Phys. C 21, L887 (1988); B. J. van Wees et al., Phys. Rev. Lett. 60, 848 (1988).

17 L. W. Molenkamp, A. A. M. Staring, C. W. J. Beenakker, R. Eppenga, C. E. Timmering, J. G. Williamson, C. J. P. M. Harmans, and C. T. Foxon, Phys. Rev. B 41, 1274 (1990). 
a)

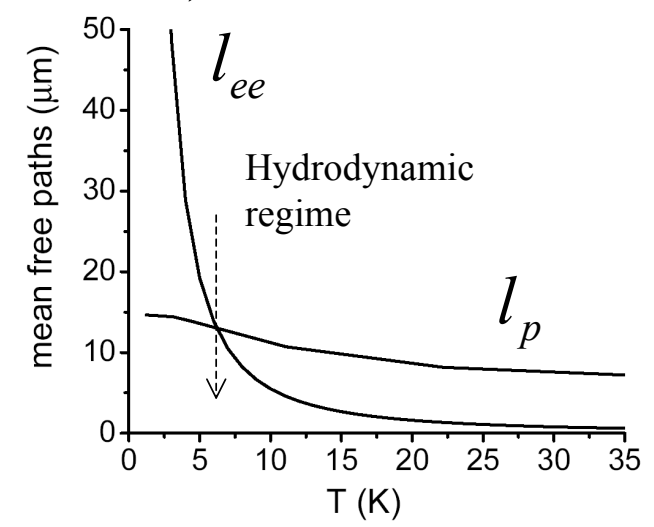

b)

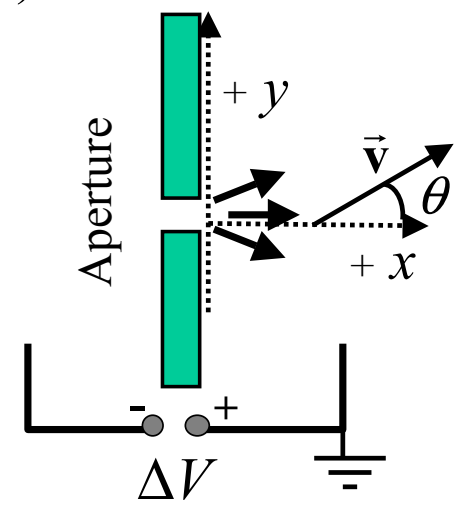

c)

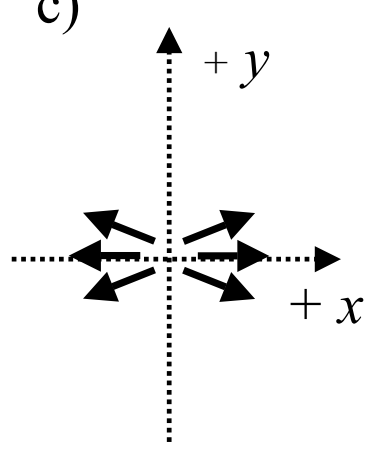

FIG. 1:

(a) Momentum relaxation and electron-electron mean free paths, $l_{p}$ and $l_{e e}$, as a function of temperature. The parameter $l_{p}$ is obtained from mobility measurements on a GaAs/AlGaAs heterostructure at the 2DES density $4.8 * 10^{11} \mathrm{~cm}^{-2}[13]$. $l_{e e}$ is calculated using eq. 14 from ref. 10 . (b) Geometry of the system with an injected electron beam. (c) Schematic of two symmetric jets in a two-dimensional plasma. 


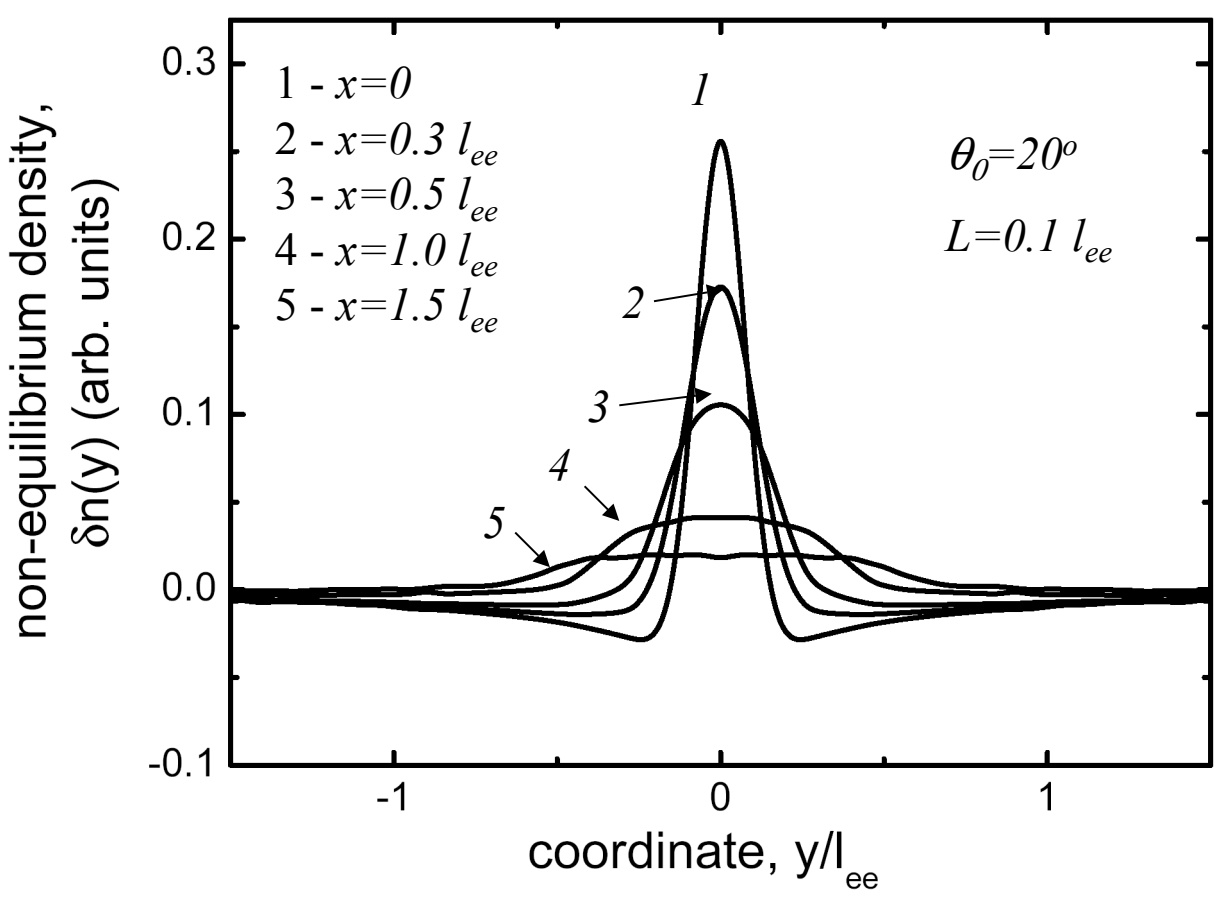

FIG. 2: Calculated non-equilibrium electron density as a function of the in-plane coordinates. 


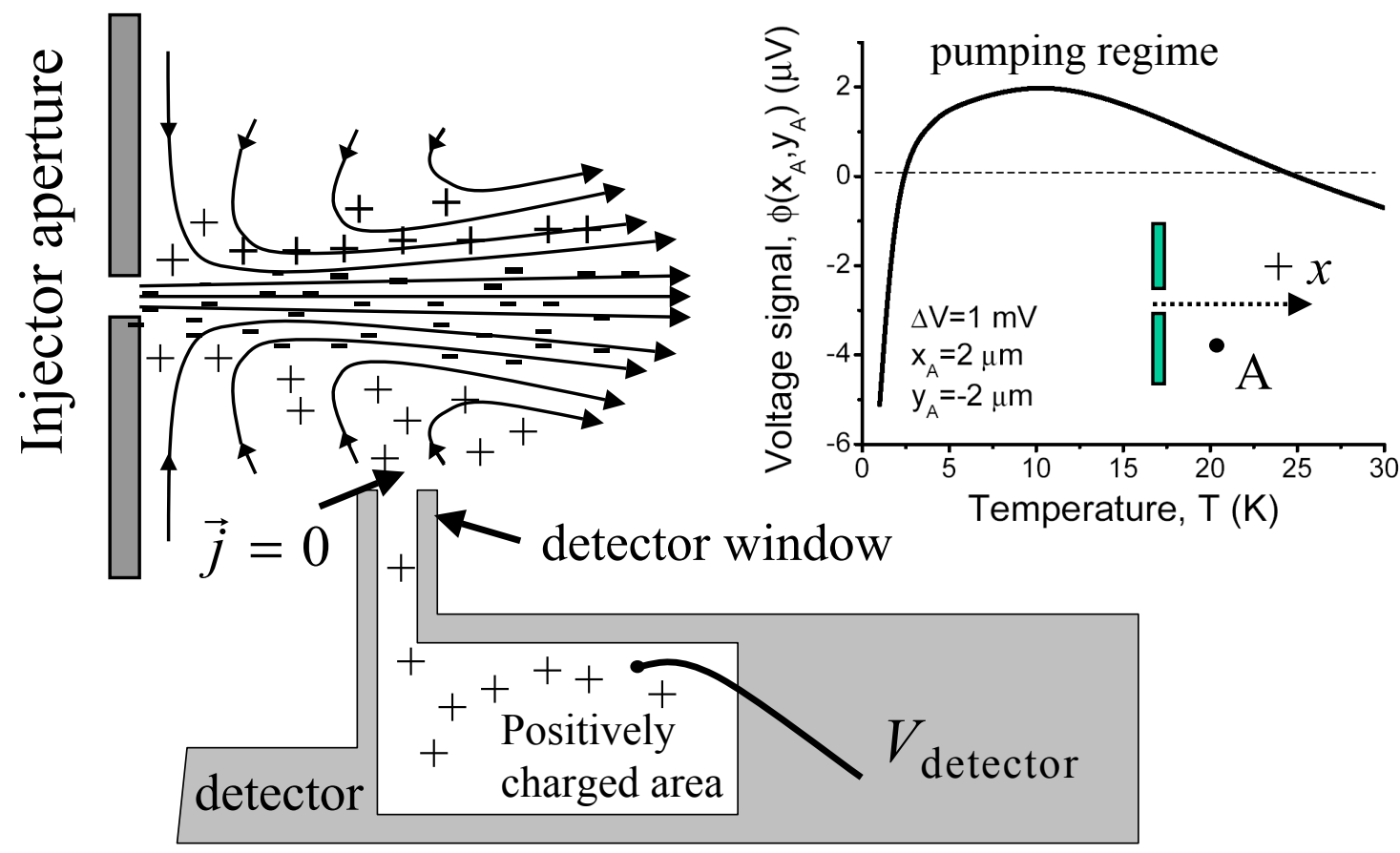

FIG. 3:

Schematic of a hydrodynamic pump in a mesoscopically patterned 2DES. The stream lines and density distribution are shown schematically. The detector serves as a probe for the potential near the main beam of electrons. Insert: The induced voltage at the point $A$ with coordinates $\left(x_{A}, y_{A}\right)$ as a function of temperature; number of conducting modes in the QPC, $N=10$; 2DES density $=$ $4.8 * 10^{11} \mathrm{~cm}^{-2}, d=400 \AA$ ค,$a_{0}^{*}=100 \AA$, and $\epsilon=12.5$. 


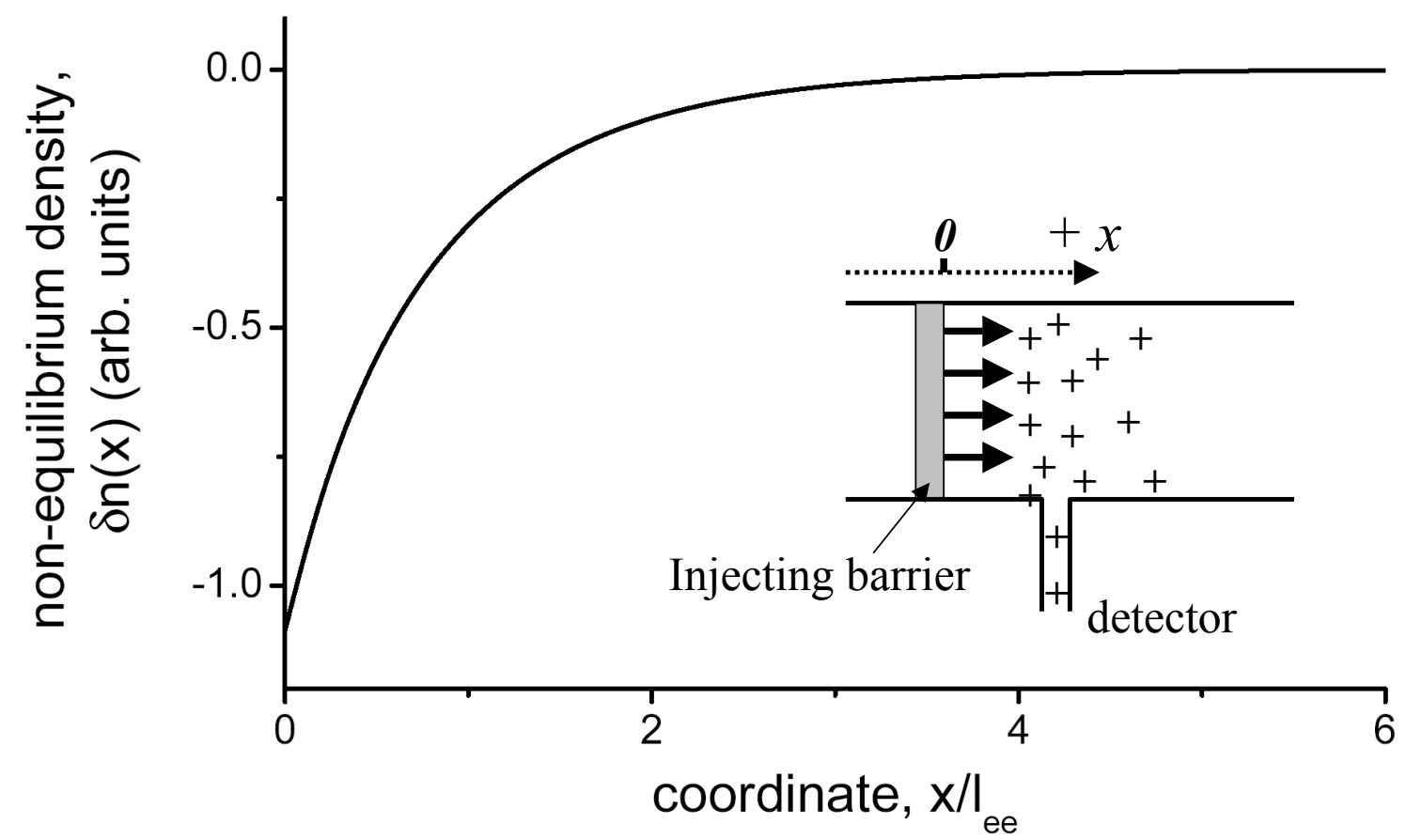

FIG. 4:

Calculated non-equilibrium electron density as a function of the $x$-coordinate in the one-dimensional system. Insert: Schematic of a hydrodynamic pump utilizing a one-dimensional channel with an injecting barrier and a detector aperture. 